\title{
Keratinocytic Malfunction as a Trigger for the Development of Solar Lentigines
}

\author{
Marjam Jeanette Barysch ${ }^{a} \quad$ Ralph Peter Braun ${ }^{a} \quad$ Isabel Kolm ${ }^{a}$ \\ Verena Ahlgrimm-Siesz $^{b}$ Rainer Hofmann-Wellenhof ${ }^{c}$ Christine Duval $^{d}$ \\ Emilie Warrick $^{d}$ Francoise Bernerd $^{d}$ Stéphanie Nouveau ${ }^{d}$ \\ Reinhard Dummer ${ }^{a}$

\begin{abstract}
${ }^{a}$ Department of Dermatology, University Hospital Zurich, Zurich, Switzerland; ${ }^{b}$ Department of Dermatology, University Hospital Salzburg, Salzburg, Austria; 'Department of Dermatology, University Hospital Graz, Graz, Austria; 'L'Oréal Research and Innovation, Aulnay sous Bois, France
\end{abstract}

\section{Keywords}

Solar lentigo · Notch1 - p53 - Keratinocytic proliferation · Senile lentigines · Lentigo solaris ·

Pigmentation

\begin{abstract}
Introduction: Solar lentigines (SL) affect chronically UV-radiated skin. Treatment is often refractory. Deeper knowledge on its pathogenesis might improve therapeutic effects. Material and Methods: Morphological characterization of 190 SL was performed and epidermal thickness, pigment distribution, dendricity, and cornification grade were measured. Immunoreactivity was investigated using Melan A, Tyrosinase, MITF, p53, and CD20, as well as Notch1 using immunofluorescence. Results: We found 2 groups of histological patterns, i.e., either acanthotic or atrophic epidermis. Lesions with basket-woven cornification and atrophic epidermis were observed in 6 out of 9 and 14 out of 16 cases from the face, respectively. Consistency of areas with a high pigmentation was observed in $96-97 \%$ of the cases. Hyperpigmentation grade and acanthosis or cornification disorders correlated positively in $88.5 \%$ of the cases. Overexpressed of $\mathrm{p} 53$ was found in 19 out of 20 lesions, presenting in a scattered distribution. A significant correlation of $\mathrm{p} 53$ and acanthosis $(p=0.003)$ and cornification grade $(p=0.0008)$ was observed. Notch1 was expressed in all SL, with the highest immunoreactivity in atrophic facial lesions. Lesions from the hands expressed Notch1 mainly in acanthotic areas with elongated rete ridges and less compact cornification. Discussion: We suggest that Notch1-dependent keratinocytic malfunction causes the development of SL. Consequently, hyperpig-
\end{abstract}


mentation would be a result and not the primary cause of the pathogenesis. Confirmation of these findings might have clinical implications as hitherto treatment has mainly focused on melanocytes and pigmentation and not on the proliferation/differentiation balance of keratinocytes.

(C) 2019 The Author(s)

Published by S. Karger AG, Basel

\section{Introduction}

Solar lentigines (SL) occur on chronically sun-exposed skin, particularly of elderly individuals [1], and clinically presents as a hyperpigmented patch. Acanthoses with elongated clubbed or branched epidermal ridges are well-known identifying histological features, usually accompanied with actinic elastosis. SL share histological and genetic features with some forms of seborrheic keratoses (SK), and an evolution of SL into SK has been suggested [2]. In the literature, there is disagreement regarding cause of SL development. Although melanocytes are only slightly increased, increased melanocytic activity has been suspected by different authors as an underlying pathogenic factor [3]. Increased melanogenic factors (endothelin 1, stem cell factor, K6F, and FGF7) and their receptors (EDNRB and c-KIT) [4], prolonged melanin removal by macrophages [5], or an abnormal increase in melanocyte stem cells in the bulge of hair follicles [6] have been proposed among others. Hence, therapy primarily targets the melanocytes and the pigment. Since these treatment modalities are refractory or at least not sustainable in most patients, the question arises of whether changes in melanocytes might be the consequence and not the cause of SL development [7].

Recent studies have provided evidence of increased expression of genes involved in the keratinocytic proliferation [8]. Keratinocytic cell growth is strictly controlled by complex signaling pathways including the tumor suppressor gene p53. UVB-induced upregulation of p53 leads to apoptosis, cell cycle arrest, and cell differentiation. Mutated p53, however, can no longer induce antiapoptosis [9]. In hyperkeratotic epithelial tumors such as actinic keratoses, Bowen disease, and squamous cell carcinoma, p53 is uniformly overexpressed [10], while it is distributed in a scattered type in SK [11]. p53 is a known activator of Notch1, which is a pivotal promoter for early keratinocytic differentiation and a preventer of keratinocytic growth. It protects keratinocytes from apoptosis by inhibition of Fox3a and has tumorsuppressive properties. Notch1 is highly expressed in well-differentiated keratinocytes [12], while it is downregulated in less differentiated keratinocytic tumor cells such as squamous cell carcinoma $[13,14]$.

In the present study, we aimed to investigate quantitative morphologic characteristics in the largest set of SL so far in order to more deeply characterize and find further indication for the pathogenesis of SL. Our results might have a clinical impact, as hitherto treatment success has been limited in a good portion of patients.

\section{Materials and Methods}

We observed 190 specimen of SL after obtaining informed consent. All lesions were diagnosed by dermosopy before shaving by dermoscopy specialists, and histology was proved by 2 different histopathologic observers. Ethical committee approval (ethical committee No. of canton Zurich: 647) was granted. Five samples of normal skin from age- and gender-matched individuals served as controls for evaluation of p53 expression. They derived from analog anatomical sites with comparable sun exposure. 
Barysch et al.: Keratinocytic Malfunction Triggers Solar Lentigines

\section{Immunohistochemistry and Immunofluorescence}

Formalin-fixed tissue samples were stained with H\&E for histological description. Masson-Fontana (MF) staining was performed for characterization of the pigmentation pattern. Immunohistochemical procedures were performed on 20 representative SL derived in equal parts from the hand and the face. Tissue samples were fixed in $4 \%$ formaldehyde overnight and embedded in paraffin. Paraffin-embedded blocks were sectioned at a 6- $\mu \mathrm{m}$ thickness and deparaffinized in xylene, hydrated with ethanol, and rinsed in distilled water. Citrate buffer incubation ( $40 \mathrm{~min}, 10 \mathrm{~mm}$ citric acid, $0.05 \%$ Tween 20, pH 6) in a steamer was used for epitope retrieval. Afterwards, sections were cooled for $20 \mathrm{~min}$ and rinsed in phosphate-buffered saline. The following primary antibodies were used: anti-melan A (1:60; Leica Microsystems), anti-tyrosinase (1:60; Novocastra), anti-MITF (clone D5, 1:60; Dako) p53 (1:60; Dako), and anti-CD20 (1:60; Dako). Notch1 immunofluorescence staining (Cell Signaling Technology) was performed on 12 of those lesions ( 5 lesions derived from the face and 7 lesions from the hand), according to a previously described method [14]. All tissue samples were microscopically observed in $\times 40$ magnification (Olympus BX51TF).

\section{Evaluation of Histology, Immunohistochemistry, and Immunofluorescence}

Cornification grade was evaluated in H\&E samples using a 3-point scale (1, predominantly basket-woven; 2 , compact orthokeratosis; and 3, thick compact cornification). The melanin distribution ( 0 , none; 1 , low; 2 , medium; and 3 , strong) and pigment amount $(0$, none; 1, low; 2, medium; and 3, strong) in each skin layer (stratum corneum, suprabasal, basal, dermal, and free dermal melanin) as well as in melanophages were evaluated via MF staining. The epidermal thickness of 3 representative areas within 1 lesion was measured with H\&E staining, scaling from the basal membrane to the stratum granulosum using Aperio Image Scope version 9.1 at a $\times 20$ magnification. Immunoreactivity to melan $\mathrm{A}$, tyrosinase, and nuclear MITF was evaluated in 20 tissues and graded as follows: 1, low; 2, medium; and 3, high. The cut-off for p53 subclassification was $5 \%$ for low and high, respectively [15], and the distribution was classified as scattered or clonal. The dendricity grade of melanocytes was measured in stainings of Melan A and tyrosinase depending on length, thickness, and amount of dendrites.

\section{Statistics}

Descriptive statistical analysis was conducted using SPSS 15.0 for Windows (SPSS Inc., Chicago, IL,USA). Pearson's $\chi^{2}$ test was used to assess the independence of epidermal thickness and cornification grade $\left(p<10^{-10}\right)$. To evaluate $p$ values in the immunohistochemistry section, a two-tailored paired $t$ test was used. $p<0.05$ was considered statistically significant. SD and 95\% CI were computed.

\section{Results}

\section{Patient Characteristics}

The 190 SL were derived from 98 patients with a mean age of 63 years (range 38.5-91.9), and $88.8 \%$ of the patients were female $(n=87)$ and $11.2 \%$ were male $(n=11)$. The lesions were derived in 165 cases from the dorsum of the hands and in 25 cases from the facial area.

\section{Histological Characterization}

The typical histology pattern was found in $96.3 \%$ of the SL matched with the typical histological patterns as described before [2]; compact cornification was present in $76.3 \%$ of the lesions ( $n=145$ ) (Fig. 1a). Lesions not revealing the typical histological pattern were derived 


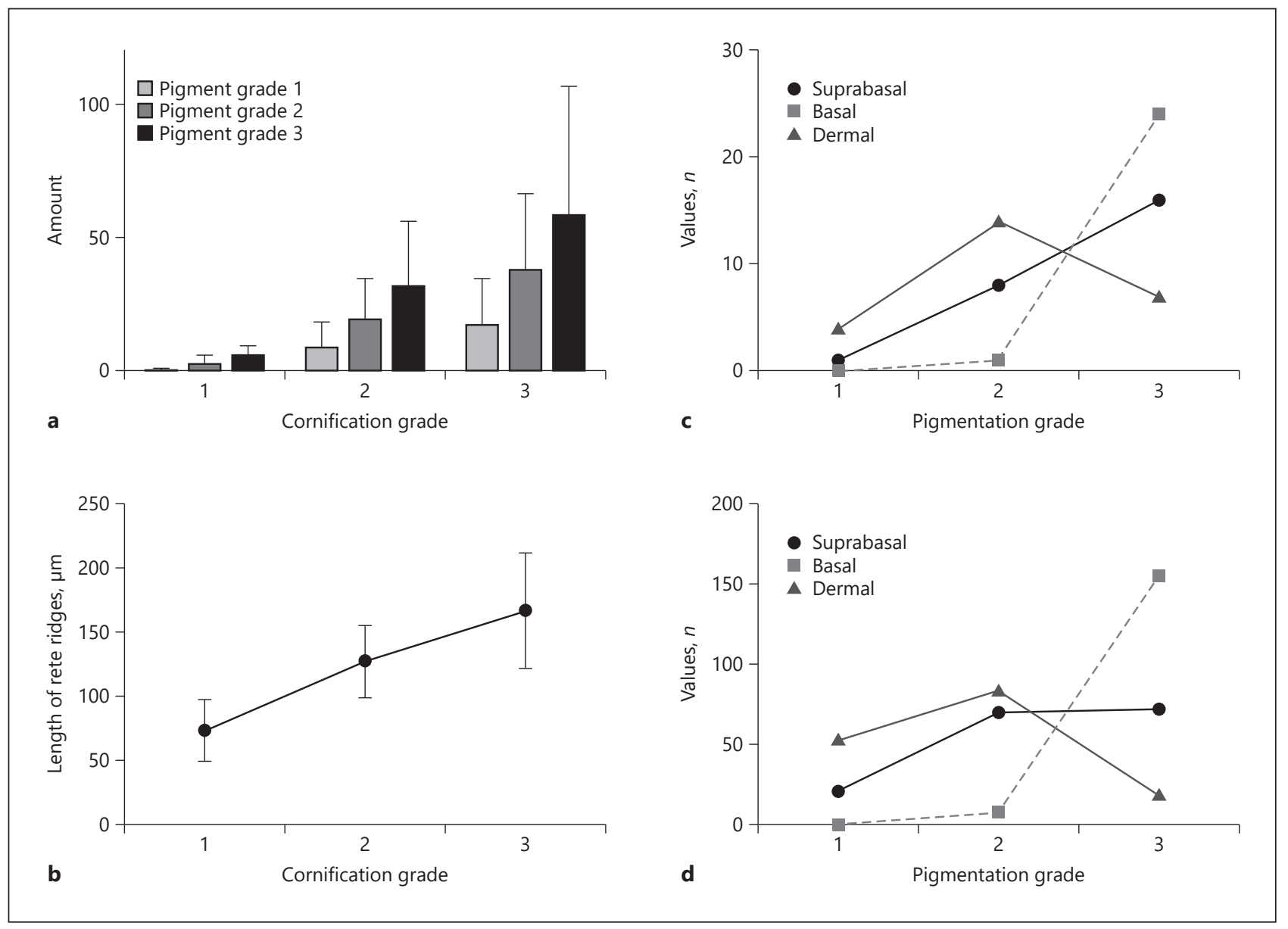

Fig. 1. a Dependency of the pigment grade (i.e., 1-3) and the cornification grade (i.e., 1-3). There was an increasing pigmentation grade with increasing cornification. b Increasing cornification grade with increasing length of the rete ridges. c Pigmentation grades in the different layers (suprabasal, basal, and dermal) of the face. Basal layers show the highest pigmentation grade, followed by suprabasal layers, while dermal layers have more moderate pigmentation grades. d Pigmentation grades in the different layers (suprabasal, basal, and dermal) of the hands. Basal layers shows the highest pigmentation grade, followed by suprabasal layers, while dermal layers have more moderate pigmentation grades.

in most cases from the face ( $n=6$, accounting for $24 \%$ of all facial lesions). Basket-woven cornification in facial SL was present in $36 \%(n=9)$ of the cases (Table 1$)$.

Atrophic epidermis was observed in 29 SL (15.3\%), and it was derived in 11 cases from the face - accounting for $56 \%$ of all facial lesions; lesions from the hands were affected in $9.1 \%$ of the cases $(n=15)$. As expected, all 190 sections showed distinctive actinic elastosis. Contradictory to Hodgon [16], disorganized cornification was commonly observed. In $6.3 \%$ of the lesions $(n=12)$, the stratum corneum revealed parakeratosis without the existence of actinic keratoses or other cofactors. Transition into seborrheic keratoses was discovered in 6 cases (3.2\%), derived in 5 cases from the hands and in 1 case from the face (Table 1 ).

A significant higher cornification grade was found in SL from the hand compared to SL from the face $(p<0.0001)$. Facial SL were furthermore significantly more atrophic than lesions from the hands $(p<0.0001)$. There was a significant positive correlation between acanthosis and cornification grade $(p<0.0001$; Fig. 1b). 
Barysch et al.: Keratinocytic Malfunction Triggers Solar Lentigines

Table 1. Morphologic characterization of solar lentigines from the hands and face

\begin{tabular}{lcccccc}
\hline & Lesions & $\begin{array}{l}\text { Typical } \\
\text { pattern }\end{array}$ & Atrophy & $\begin{array}{l}\text { Actinic } \\
\text { elastosis }\end{array}$ & $\begin{array}{l}\text { Parakera- } \\
\text { tosis }\end{array}$ & $\begin{array}{l}\text { Transition } \\
\text { into SK }\end{array}$ \\
\hline Hands & 165 & 164 & 15 & 165 & 11 & 5 \\
Face & 25 & 19 & 14 & 25 & 1 & 1 \\
\hline Total & 190 & 183 & 29 & 190 & 12 & 6 \\
\hline
\end{tabular}

Values are presented as numbers of solar lentigines. SK, seborrheic keratosis.

\section{Pigment Distribution}

Overall, the pigmentation of facial lesions was significantly higher than in SL from the hands $(p=0.024)$. Pigment distribution differed significantly within the different layers $(p<0.0001)$, with the highest pigmentation grade in basal layers (mean: 2.95), followed by suprabasal (mean: 2.35) and dermal layers (mean: 1.83). Basal layers presented the most highly pigmented areas in each cut, with $95.2 \%$ of them graded as highly pigmented $(n=$ $179)$ and $4.8 \%$ of them classified as medium pigmented $(n=9)$. None of them were graded as low pigmented. Suprabasal layers were partly highly or medium pigmented in $46.8 \%$ $(n=88)$ and $41.5 \%(n=78)$ of the cases, respectively; only $11.7 \%(n=22)$ of the cases revealed a low pigmentation. The pigmentation amount in the dermis was low in $31.5 \%$ $(\mathrm{n}=57)$, medium in 54.1\% ( $\mathrm{n}=98)$, and high in $14.4 \%(\mathrm{n}=26)$ of the cases. The upper dermis showed a few melanophages, free melanin, and inflammatory infiltration, while the deeper reticular dermis remained unaffected. Within the lesions, less pigmented areas were noticed (Fig. 1c, d).

Central regions of appendices such as the excretory ducts were pigment free, whereas their margins to the surrounding epidermis were intensely pigmented. Pigmentation in the periphery of the eccrine gland epithelium was more intense than in the hair follicle, and it extended further into the center. The epithelium of the hair follicles was pigment free in $32.4 \%(n=23)$ of the cases and slightly pigmented at the margins in $36.6 \%(n=26)$, with lower melanin levels than the surrounding tissue; $29.6 \%(n=21)$ revealed the same pigmentation at the margin as the surrounding tissue, and only 1 case $(1.4 \%)$ showed higher pigmentation at the margin than in the surrounding tissue (Fig. 2a).

In $56.0 \%(n=51)$ of the lentigines with eccrine ducts within the tissue, the eccrine ducts showed a similar epithelial pigment distribution compared to the surrounding tissue; in $34.1 \%(n=31)$ there was less and in $6.6 \%(n=6)$ there was no pigmentation. Only 3 lesions $(3.3 \%)$ were more strongly pigmented in the epithelium of the eccrine ducts. The cornification above the orifices was basket woven and not compact; furthermore, there was no hyperpigmentation. The surrounding area, however, revealed a compact and highly pigmented cornification. Both areas were sharply demarcated from each other. The mean pigmentation grade of eccrine glands was slightly lower than the pigmentation of full lesions (1.56 vs. 1.74), whereas the epithelium of the hair follicles was distinctly less pigmented than the surrounding tissue (mean pigmentation grade: 1.0).

The pigment amounts in basal and suprabasal layers were consistent. Areas with highly pigmented basal layers showed also a high pigmentation of the upper epidermal layers in the stratum corneum in $97.4 \%(n=186)$ and in the dermis in $96.3 \%(n=184)$ of the cases based on the mean of melanophages. Within 1 lesion, areas with highly disordered cornification revealed a higher pigmentation than the surrounding areas or areas with less compact cornification in 171 cases (89.53\%). Likewise, areas with abnormal elongation of rete ridges also 


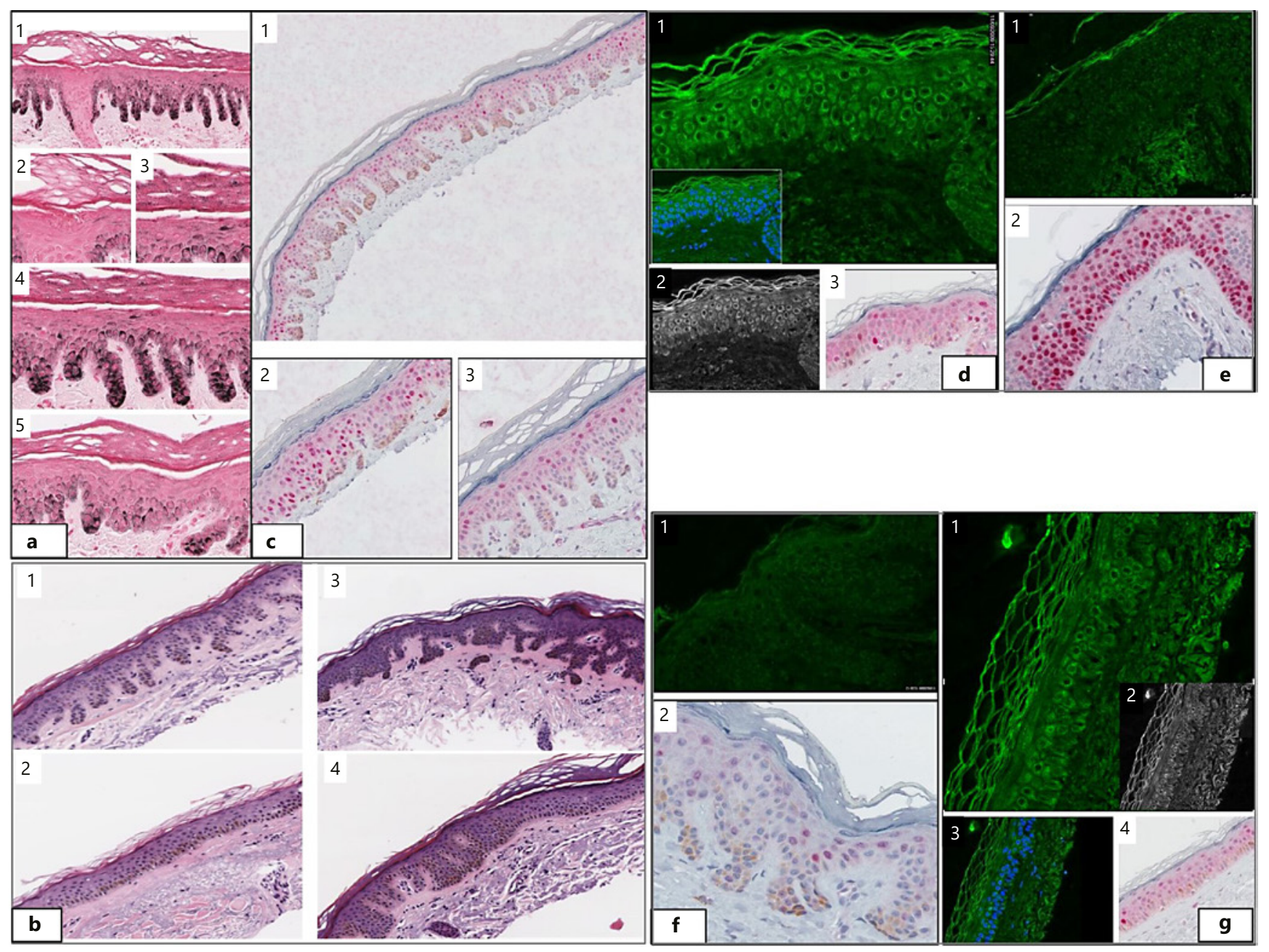

Fig. 2. a Masson-Fontana staining of a solar lentigo revealing compact cornification above elongated rete ridges and tighter cornification above areas of deep and intense pigment. Less compact cornification above the excretory duct (a1); stratum corneum above the orifice (a2) and above the area with a high basal and suprabasal pigmentation (a3); higher and denser pigment in stratum corneum above areas with a high basal pigmentation (a4), and lower pigment in stratum corneum above rete ridges with a lower pigmentation (a5). b Solar lentigines in H\&E staining. b1 Typical histological pattern showing elongated rete ridges and compact cornification and distinct actinic elastosis. b2 Atrophic solar lentigo derived from the face. b3 Solar lentigo with transformation into seborrheic keratosis. b4 Area with elongated rete ridges showing a higher compact cornification than the area with more atrophic epidermis. c p53 overexpression with a scattered distribution. There was a higher expression in areas with compact cornification and a lower expression in areas with less compact cornification. $\mathbf{d}$ Notch1 expression in a lesion derived from the face with a more atrophic epidermis and basket-woven cornification with Hoechst 6244 nuclear counterstaining (d1), blackwhite filter (d2), and p53 reactivity of the same lesion (d3). e p53 and Notch1 expression in an atrophic area with more compact cornification of a solar lentigo of the face. $\mathbf{f}$ Solar lentigo of the dorsum of a hand with elongated rete ridges and compact cornification with p53 and Notch1 positivity. g Atrophic lesion deriving from the dorsum of the hand with basket-woven cornification; a higher Notch1 expression was found on the right end with an elongated rete ridge (g1), black-white (g2), nuclear counterstaining with Hoechst 6244 (g3), and p53 (g4). 
Barysch et al.: Keratinocytic Malfunction Triggers Solar Lentigines

Table 2. Insensitivity of pigment distribution and immunoreactivity to melan A, tyrosinase, and MITF by histologic layer

\begin{tabular}{lcccc}
\hline & Suprabasal layer & Basal layer & Dermis & Appendices \\
\hline Masson-Fontana stain & $0.9 \pm 0.8$ & $2.6 \pm 0.5$ & $3.0 \pm 0.2$ & $1.9 \pm 0.6$ \\
Melan A & $1 \pm 0$ & $2 \pm 0.5$ & $1.75 \pm 0.5$ & $1.8 \pm 0.4$ \\
MITF & $1.7 \pm 0.4$ & $1.9 \pm 0.5$ & $1.3 \pm 0.5$ & $1.4 \pm 0.6$ \\
TYR & $1.7 \pm 0.5$ & $2.7 \pm 0.5$ & $1 \pm 0$ & \\
p53 $>5 \%$ & $2.2 \pm 0.7$ & & & \\
\hline
\end{tabular}

Values are presented as means \pm SD.

showed a higher pigment distribution than areas within the lesion with lower elongated rete ridges $(88.5 \%, n=169$; Fig. 2a, b).

\section{Immunohistochemistry for Melanocytic Markers}

Melan A immunoreactivity of all of the observed lesions was not only observed among the basal layer as described before [17] but also in melanosomes of the suprabasal layers, the melanophages of the dermis, and the appendages such as pilary canals and eccrine ducts. Therefore, nuclear reactivity for MITF and cytoplasmic reactivity for tyrosinase were used for verification of the melanocytes. There was a strong consistency of immune reactivity for tyrosinase, MITF, and melan A, particularly in the basal layers of all 20 tissues. The dendricity grade was graded as medium in 65\% (13/20), low in 20\% ( $n=4)$, and high $(n=3)$ in $15 \%$ of the cases. The tumor suppressor gene p53 was overexpressed ( $>5 \%$ of all keratinocytes) in the suprabasal layer and - to a lower degree - in the basal layer in 19 out of 20 lesions. p53 was presented in a scattered, not a clonal, cell distribution, and it was more highly expressed in particular areas within the lesions: p53 expression and acanthosis (length of the rete ridges) correlated significantly in all lesions overall $(p=0.0028)$, and also in lesions particularly of the face $(p=0.0315)$ or the hands $(p=0.0426)$. Cornification grade correlated significantly with p53 reactivity ( $p=0.0008)$ overall and particularly SL of the hands $(p=0.0031)$, but not face alone ( $p=0.5588$; Table 2; Fig. 2 c). Age-matched controls revealed the absence of p53 expression in $80 \%$ of the cases; a scattered distribution, with less than $5 \%$ of the cells affected, was found in $20 \%$ of the age-matched controls.

\section{Immunofluorescence}

Notch1 was expressed in all observed 13 SL in suprabasal layers compared to control skin. The highest expression was found in atrophic lesions with basket-woven cornification deriving from the face. Lesions of the hands were more intensively immunoreactive to Notch1 in acanthotic areas with elongated rete ridges and less compact cornification; areas with a highly compact cornification or atrophic epidermis were less reactive. Colocalization of Notch1 and p53 in the same area was not detected consistently (Table 2; Fig. 2c-f).

\section{Discussion}

Various past authors supposed melanocytes to be the cause of SL development. They found an increased number of melanocytes $[3,6]$, increased melanogenic factors and their receptors $[4,18,19]$, prolonged melanin removal by macrophages, or an abnormal increase in melanocyte stem cells in the bulge of hair follicles [6] to be the main causative factors [5, 
8]. Accordingly, and consistent with the main clinical characteristic of SL which is hyperpigmentation, treatment targets predominantly melanocytes melanogenesis. As this approach does not respond satisfactorily in most cases, assumption regarding the pathogenesis seems reasonable.

Different authors could not find pigmentation genes differentially expressed in SL. Instead, genes which are involved in the keratinization process, such as SPRR4 [20] or LCE1E were detected, and sciellin was proved to be downregulated [20,21]. Cario-Andre et al. [22] proposed retaining perilesional pigment-harboring keratinocytes as a causative factor.

Histological similarities between SL and SK and clinical observations suggesting transformation of SL into reticular-acanthotic types of SK point to an underlying keratinocytic process in the development of SL [23]. With the observation of abnormal high levels of KRT5, KRT 10, and keratinocyte growth factor (receptor), an important role of basal KC proliferation has been demonstrated by different authors [8, 24, 25]. Correspondingly, similar mutations in both SL and SK such as FGFR3 and PIK3CA have been detected [26, 27], supporting the hypothesis that SL and SK might evolve from the same spectrum. However, only a small percentage of SL are positive for SK mutations (17\% for FGFR3 and 7\% for PIK3CA) [27]. This raises the difficulty of clinically distinguishing SL from SK and the fact that some hyperpigmented lesions could be mixed SL-SK.

In the presented study, we aimed to morphologically study SL accurately in order to find deeper morphologic evidence for the development of SL. To our knowledge, this is the greatest set of investigated SL containing 190 specimens. We identified a greater proportion of facial SL with a rather atrophic epidermis than those presenting the commonly known elongated rete ridges. Of note, these lesions were dermoscopically proven to be SL by a dermoscopic specialist before shaving; actinic keratosis or other entities could be excluded by dermoscopy and by histology. We further investigated inhomogeneous pigmentation throughout SL, sparing the central regions of the eccrine ducts, while their edges were intensely hyperpigmented. Also the hair follicles were slightly or not pigmented at all in most cases. The periphery of the eccrine ducts was more intensely pigmented than the periphery of the hair follicles. We can only speculate about the underlying reason for and result of the spared pigmentation and the absent cornification disorders in the central appendices, but it seems that adnexal structures are somehow not affected by the pathologic dysfunction. To our knowledge, these morphologic findings have not been described elsewhere so far.

We found consistency of areas with a high pigmentation in the basal and suprabasal layers as well as in dermal layers in most cases ( 97.4 and $96.3 \%$, respectively). We further noted a clear relation between pigmentation intensity and acanthosis grade in $88.5 \%$ as well as cornification disorders within one lesion. The melanocytic origin of the cells was verified by the immunoreactivity of melan A, tyrosinase, and nuclear MITF. Contrary to SK, which show scattered homogenous nuclear p53 expression throughout the complete lesion $[11,28]$, we found a focally more pronounced - though nonclonal - overexpression of p53 in suprabasal and basal layers of SL in areas of stronger compact cornification and acanthosis. Their distribution patterns also varied depending on the localization; facial lesions showed unstructured distributions. Lesions of the hands, however, presented a higher p53 expression in areas with acanthosis with elongated rete ridges and in areas with compact cornification. Interestingly, an abnormal pigment distribution was more intense in the surrounding areas of p53 overexpression and not within the overexpressed lesion. We found a significant positive correlation between expression of the tumor suppressor p53 and acanthosis grade $(p=0.003)$ as well as cornification grade $(p=0.0008)$. Accordingly, Notch1 showed different distributions in the face and on the hands; while SL of the face were - independently of the cornification grade - Notch1 reactive in atrophic and in acanthotic lesions, SL of the hands showed a higher reactivity in areas with acanthosis and elongated rete ridges. These observa- 
Barysch et al.: Keratinocytic Malfunction Triggers Solar Lentigines

tions underlie a p53- and Notch1-dependent keratinocytic dysfunction possibly caused by a reactive p53 upregulation in order to regulate further neoplastic proliferation. Impairment of the proliferation/differentiation program in keratinocytes could explain the retention of pigment in the basal layer, subsequently leading to an increased amount of melanin in the suprabasal layers and finally in the stratum corneum. The accumulation of melanin in the epidermis might trigger the activation of dermal melanophages, which is illustrated by a stronger dermal pigment in areas with a high pigmentation. This model supports the thesis that hyperpigmentation is a result of the development SL and not the underlying cause as believed by various authors [22, 23, 29].

A limitation of this study is the semiquantitative immunohistochemical observational setting. Hypothetical findings would have to be confirmed on transcriptional levels. However, this study has the largest number of lesions, and new histological patterns have been found.

\section{Conclusions}

To conclude, in this preliminary study, we assume defective keratinocytic control mechanisms in the affected areas to be the underlying cause of the clinical pattern of SL. Adnexal structures might not respond to those mechanisms as at least their centers are not pigmented and/or not affected by cornification disorders. Confirmation of the presented results might have a fundamental impact on novel therapy lines, as hitherto treatment targeting pigmentation has treated symptomatic targets and not causative ones.

\section{Statement of Ethics}

Subjects have given their written informed consent. The study protocol was approved by the research institute's committee on human research. This research was conducted ethically in accordance with the World Medical Association Declaration of Helsinki.

\section{Disclosure Statement}

Christine Duval, Emilie Warrick, Francoise Bernerd, and Stéphanie Nouveau are employees of L'Oréal. Prof. Dummer has intermittent, project-focused consulting and/or advisory relationships with Novartis, Merck Sharp \& Dohme (MSD), Bristol-Myers Squibb (BMS), Roche, Amgen, Takeda, Pierre Fabre, and Sun Pharma outside of the submitted work. Otherwise we have no further disclosure statements.

\section{Funding Sources}

This work was supported by the Connaissances and Performances Department - Advanced Research L'Oréal Recherche and Innovation. 


\section{Author Contributions}

Marjam Jeanette Barysch, Christine Duval, Emilie Warrick, Francoise Bernerd, Stéphanie Nouveau, and Reinhard Dummer made substantial contributions to the conception or design of this work.

Marjam Jeanette Barysch contributed substantially to acquisition of data for and drafting of this work.

Marjam Jeanette Barysch, Ralph Braun, Isabel Kolm, Verena Ahlgrimm-Siesz, Rainer Hofmann-Wellenhoff, and Reinhard Dummer made substantial contributions to analysis and interpretation of data for this work.

Ralph Braun, Isabel Kolm, Verena Ahlgrimm-Siesz, Rainer Hofmann-Wellenhoff, Christine Duval, Emilie Warrick, Francoise Bernerd, Stéphanie Nouveau, and Reinhard Dummer revised this work critically for important intellectual content.

All of the authors gave final approval of the version to be published and agreed to be accountable for all aspects of this work in ensuring that questions related to the accuracy or integrity of any part of this work are appropriately investigated and resolved.

\section{References}

1 Bastiaens M, Hoefnagel J, Westendorp R, Vermeer BJ, Bouwes Bavinck JN. Solar lentigines are strongly related to sun exposure in contrast to ephelides. Pigment Cell Res. 2004 Jun;17(3):225-9.

2 Andersen WK, Labadie RR, Bhawan J. Histopathology of solar lentigines of the face: a quantitative study. J Am Acad Dermatol. 1997 Mar;36(3 Pt 1):444-7.

3 Praetorius C, Sturm RA, Steingrimsson E. Sun-induced freckling: ephelides and solar lentigines. Pigment Cell Melanoma Res. 2014 May;27(3):339-50.

4 Kovacs D, Cardinali G, Aspite N, Cota C, Luzi F, Bellei B, et al. Role of fibroblast-derived growth factors in regulating hyperpigmentation of solar lentigo. Br J Dermatol. 2010 Nov;163(5):1020-7.

5 Watanabe T, Tahira M, Morino S, Horie T, Adachi K, Tsutsumi R, et al. Novel morphological study of solar lentigines by immunohistochemical and electron microscopic evaluation. J Dermatol. 2013 Jul;40(7):528-32.

6 Yamada T, Hasegawa S, Inoue Y, Date Y, Arima M, Yagami A, et al. Comprehensive analysis of melanogenesis and proliferation potential of melanocyte lineage in solar lentigines. J Dermatol Sci. 2014 Mar;73(3):251-7.

7 Imhof L, Dummer R, Dreier J, Kolm I, Barysch MJ. A Prospective Trial Comparing Q-Switched Ruby Laser and a Triple Combination Skin-Lightening Cream in the Treatment of Solar Lentigines. Dermatol Surg. 2016 Jul; 42(7):853-7.

8 Choi W, Yin L, Smuda C, Batzer J, Hearing VJ, Kolbe L. Molecular and histological characterization of age spots. Exp Dermatol. 2017 Mar;26(3):242-8.

9 Muller PA, Vousden KH. Mutant p53 in cancer: new functions and therapeutic opportunities. Cancer Cell. 2014 Mar;25(3):304-17.

10 Sakiz D, Turkmenoglu TT, Kabukcuoglu F. The expression of p63 and p53 in keratoacanthoma and intraepidermal and invasive neoplasms of the skin. Pathol Res Pract. 2009;205(9):589-94.

11 Ko CJ, Shintaku P, Binder SW. Comparison of benign keratoses using p53, bcl-1, and bcl-2. J Cutan Pathol. 2005 May;32(5):356-9.

12 Mandinova A, Lefort K, Tommasi di Vignano A, Stonely W, Ostano P, Chiorino G, et al. The FoxO3a gene is a key negative target of canonical Notch signalling in the keratinocyte UVB response. EMBO J. 2008 Apr;27(8): 1243-54.

13 Dotto GP. Notch tumor suppressor function. Oncogene. 2008 Sep;27(38):5115-23.

14 Kolev V, Mandinova A, Guinea-Viniegra J, Hu B, Lefort K, Lambertini C, et al. EGFR signalling as a negative regulator of Notch1 gene transcription and function in proliferating keratinocytes and cancer. Nat Cell Biol. 2008 Aug;10(8):902-11.

15 Hussein MR, Al-Badaiwy ZH, Guirguis MN. Analysis of p53 and bcl-2 protein expression in the non-tumorigenic, pretumorigenic, and tumorigenic keratinocytic hyperproliferative lesions. J Cutan Pathol. 2004 Nov; 31(10):643-51.

16 Hodgson C. Senile lentigo. Arch Dermatol. 1963 Feb;87(2):197-207.

17 Helm K, Findeis-Hosey J. Immunohistochemistry of pigmented actinic keratoses, actinic keratoses, melanomas in situ and solar lentigines with Melan-A. J Cutan Pathol. 2008 Oct;35(10):931-4.

18 Hattori H, Kawashima M, Ichikawa Y, Imokawa G. The epidermal stem cell factor is over-expressed in lentigo senilis: implication for the mechanism of hyperpigmentation. J Invest Dermatol. 2004 May;122(5):1256-65. 
19 Kadono S, Manaka I, Kawashima M, Kobayashi T, Imokawa G. The role of the epidermal endothelin cascade in the hyperpigmentation mechanism of lentigo senilis. J Invest Dermatol. 2001 Apr;116(4):571-7.

20 Goyarts E, Muizzuddin N, Maes D, Giacomoni PU. Morphological changes associated with aging: age spots and the microinflammatory model of skin aging. Ann N Y Acad Sci. 2007 Nov;1119(1):32-9.

21 Warrick E, Duval C, Nouveau S, Bastien P, Piffaut V, Chalmond B, et al. Morphological and molecular characterization of actinic lentigos reveals alterations of the dermal extracellular matrix. Br J Dermatol. 2017 Dec; 177(6):1619-32.

22 Cario-Andre M, Lepreux S, Pain C, Nizard C, Noblesse E, Taïeb A. Perilesional vs. lesional skin changes in senile lentigo. J Cutan Pathol. 2004 Jul;31(6):441-7.

23 Mehregan AH. Lentigo senilis and its evolutions. J Invest Dermatol. 1975 Nov;65(5):429-33.

24 Chen N, Hu Y, Li WH, Eisinger M, Seiberg M, Lin CB. The role of keratinocyte growth factor in melanogenesis: a possible mechanism for the initiation of solar lentigines. Exp Dermatol. 2010 Oct;19(10):865-72.

25 Lin CB, Hu Y, Rossetti D, Chen N, David C, Slominski A, et al. Immuno-histochemical evaluation of solar lentigines: the association of KGF/KGFR and other factors with lesion development. J Dermatol Sci. 2010 Aug; 59(2):91-7.

26 Hafner C, López-Knowles E, Luis NM, Toll A, Baselga E, Fernández-Casado A, et al. Oncogenic PIK3CA mutations occur in epidermal nevi and seborrheic keratoses with a characteristic mutation pattern. Proc Natl Acad Sci USA. 2007 Aug;104(33):13450-4.

27 Hafner C, Stoehr R, van Oers JM, Zwarthoff EC, Hofstaedter F, Landthaler M, et al. FGFR3 and PIK3CA mutations are involved in the molecular pathogenesis of solar lentigo. Br J Dermatol. 2009 Mar;160(3):546-51.

28 Kamiya M, Takeuchi Y, Katho M, Yokoo H, Sasaki A, Nakazato Y. Expression of p73 in normal skin and proliferative skin lesions. Pathol Int. 2004 Dec;54(12):890-5.

29 Montagna W, Hu F, Carlisle K. A reinvestigation of solar lentigines. Arch Dermatol. 1980 Oct;116(10):1151-4. 